DÉCOLONISATION ET CONSTRUCTION NATIONALE AFRIQUE, ASIE ET QUÉBEC

TITRE: UNE ÉQUIPE, UN PAYS : LA RÉCONCILIATION RACIALE POST APARTHEID AUTOUR DU RUGBY SUDAFRICAIN

Auteur(s): Marie-Claude BeAuregard, Université De Sherbrooke

Publication: Décolonisation et construction nationale: Afrique, Asie et Québec

PAGE: 155-168.

Directeurs: PATrick Dramé, PAScal SCALlon-Chouinard et FrançoISE NozATI

ÉditeuR: LES ÉDITIONS DE L'UNIVERSITÉ DE SHERBROOKE, 2016.

ISBN: 978-2-7622-0354-7

URI: HTTP://HDL.HANDLE.NET/11143/8767

DOI: HTTP://DX.DOI.ORG/10.17118/11143/8767 


\title{
UNE ÉQUIPE, UN PAYS : LA RÉCONCILIATION RACIALE POST APARTHEID AUTOUR DU RUGBY SUD-AFRICAIN
}

\author{
Marie-Claude Beauregard
}

Durant des années, l'Afrique du Sud est soumise à l'apartheid, un système légal de ségrégation raciale qui dicte la nature des rapports sociaux au sein du pays. De 1948 jusqu'au début des années 1990, la population noire vit au rythme des politiques inégalitaires des Afrikaners blancs, ces Sud-Africains descendants des colons néerlandais qui s'installent au Cap de Bonne Espérance dès 1652. La nation dont hérite Nelson Mandela en 1994, au sein du premier gouvernement démocratiquement élu, demeure fortement divisée sur le plan racial, traumatisée par des années de régime autoritaire et raciste. Les rapports Noirs/Blancs n'étant plus régis par des législations et un État policier, la question de la cohabitation se pose dorénavant.

L'élection du premier président noir du pays réjouit les Noirs, mais inquiète les Blancs, effrayés par la possibilité de représailles violentes. Mandela a cependant une vision très précise de la manière dont la cohabitation raciale doit se faire au sein de la " nation arc-enciel ». II entend réunifier pacifiquement la population à travers divers symboles chers aux Afrikaners : parmi ceux-ci, l'équipe nationale de rugby, les Springboks, véritable emblème du nationalisme afrikaner et symbole de l'oppression pour la majorité noire. Mandela considère cependant qu'elle fait partie du patrimoine national et de ce fait, est la propriété de la nation entière. Dès son arrivée au pouvoir en date du 10 mai 1994, il saisit l'importance significative de ce sport pour les Afrikaners.

C'est ainsi qu'il tente d'utiliser le rugby et sa symbolique afın de parvenir à une réunification raciale pacifique. On peut donc se questionner sur comment et pourquoi le rugby sud-africain devient, dans ce contexte de tensions raciales post apartheid, le vecteur principal de la réconciliation nationale? Pourquoi un sport et une équipe symbolisant l'apartheid contribuent-ils à réunir les Noirs et les Blancs de façon pacifique? À la sortie de l'apartheid, la question de la politique générale du nouveau gouvernement et des modalités de la gestion de l'après-apartheid se pose. Nous tenterons de cerner et d'expliquer l'attitude adoptée par Mandela envers ses compatriotes blancs et en quoi le rugby plutôt que le football, sport le plus populaire en Afrique, devient un élément central de la réconciliation nationale. À ce stade, il est possible d'affirmer qu'après le démantèlement du régime de l'apartheid, la réconciliation nationale sud-africaine s'effectue autour du rôle joué par Nelson Mandela dans l'organisation 
de la Coupe du monde de rugby de 1995. Le présent travail s'appuie sur le film Invictus, du réalisateur américain Clint Eastwood. Produit en 2009 et sorti dans les salles en 2010, le long métrage met en vedette Morgan Freeman et Matt Damon dans les rôles principaux de Nelson Mandela et du capitaine sud-africain François Pienaar. Si le film est réalisé sur des bases crédibles et rend bien la réalité historique, certaines critiques peuvent être formulées à commencer par l'occultation de la part d'Eastwood de plusieurs sujets tels que la relation entre Mandela et sa fille, la relation entre Pienaar et sa famille.

D'ailleurs, il écarte également certains personnages importants comme Pienaar pour se concentrer uniquement sur la figure du président Mandela au point de produire une œuvre presque hagiographique selon les critiques de Jacques Mandelbaum ${ }^{1}$. Marie Corrigall relève le fait que le long métrage ne traite pas des différents problèmes de cohabitation et de réconciliation qui secouent l'Afrique du Sud après la Coupe du monde. II s'agit selon elle, d'un film destiné à un public américain avec un dénouement hollywoodien². II importe donc, à la lumière du visionnement et des différentes critiques, d'être vigilant puisqu'il s'agit ici d'un récit adapté dans le cadre d'un film et non pas de documents d'archives classiques : la nécessité de distinguer les éléments historiques des éléments fictifs est primordiale.

Des thèmes majeurs peuvent être relevés suite au visionnement du film : la réconciliation et le pardon autour de la figure de Mandela, les Springboks et Peinaar, la valorisation des symboles nationaux à travers le maillot et les couleurs de l'équipe et l'importance de l'hymne national. Parallèlement à cela, certains extraits tirés de Un long chemin vers la liberté, les mémoires de Nelson Mandela parus en 1995, seront utilisés dans le but de souligner la position du président sud-africain. Le premier chapitre présente le contexte sud-africain, des origines de l'apartheid aux négociations entourant la sortie de crise et les enjeux qui attendent la nouvelle nation démocratique. Le deuxième chapitre traite des thèmes de la réconciliation et du pardon autour de Mandela et de l'attitude adoptée par ce dernier pour unifier pacifiquement le pays. Enfin, le dernier chapitre traite du rugby en tant que tel, de la culture sportive reliée à cette discipline et de l'équipe des Springboks comme vecteur d'identité.

1. Jacques Mandelbaum, «Invictus: réconciliation raciale sur une pelouse de rugby», Le Monde, (12 janvier 2010), [en ligne] http://www.lemonde.fr/cinema/article/2010/01/12/invictus-reconciliation-raciale-sur-une-pelouse-de-rugby_1290640_3476.html, page consultée le 9 mai 2013.

2. Marie Corrigall, "Mandela revu et corrigé dans Invictus», Courrier international, (12 janvier 2010), [en ligne] http://www.courrierinternational.com/article/2010/01/12/mandela-revu-et-corrige-dans-invictus, page consultée le 9 mai 2013. 


\section{Whites only: Le RÉGIME de L'APARTHeid en Afrique du Sud}

DE LA DISCRIMINATION RACIALE AU SYSTĖME LÉGAL DE SÉPARATION : LES ORIGINES DE L'APARTHEID

Le 30 juin 1991, l'Afrique du Sud tourne une page importante de son histoire en abolissant définitivement les structures racistes de l'apartheid, régime inégalitaire instauré en 1948. Dans la langue afrikaans, le terme « apartheid » signifie séparation, mise à l'écart. Suite aux deux guerres mondiales, le terme est vulgarisé par le langage politique et désormais, on parlera du développement séparé de chaque « race ». Fondé sur une prétendue supériorité des Blancs par rapport aux Noirs, l'apartheid s'applique aussi à d'autres groupes ethniques qui composent l'Afrique du Sud tels que les Métis et les Indiens. Le régime de l'apartheid sud-africain est précédé du Colour Bar (la barrière de couleur), une doctrine coloniale britannique appliquée dans les colonies de l'Empire qui opère une discrimination raciale au sein des groupes ethniques, sans pour autant légiférer sur les relations interraciales ${ }^{3}$.

C'est cependant en 1948 que le terme « apartheid » apparaît dans le langage politique sud-africain avec l'arrivée au pouvoir du Parti national purifié de l'ultranationaliste Daniel Malan. Ce mouvement revendique une séparation raciale radicale entre les Blancs et les non-Blancs. Initialement, l'apartheid est un concept utilisé dans le but de rallier l'électorat blanc extrémiste : il devient peu à peu la base du régime politique. Mais alors comment une poignée de Blancs parviennent-ils à exercer un tel contrôle sur une majorité noire? La minorité blanche colonisatrice, détentrice des rênes du pouvoir en Afrique du Sud, met sur pied un système politique totalitaire garant de cette hégémonie des Blancs et qui, légalement, opère une séparation marquée entre les deux « races ». Cette mise en place d'un racisme légal s'effectue sous l'égide du ministre des Affaires indigènes, Hendrik Verwoerd. À partir des années 50, une série de mesures juridiques sont prises dans cette optique de séparation ${ }^{4}$. En 1953, avec le Reservation of Separate Amenities Act, le gouvernement opère un apartheid géographique en prévoyant des lieux et des équipements séparés pour chaque groupe. On prohibe même les relations sexuelles entre personnes de couleurs différentes avec l'Immorality Act de 1950. Il y a donc véritablement un désir de contrôle total de la part des législateurs. Une ségrégation urbaine se met également en place : on « parque » les populations noires en périphérie de la ville, dans des townships défavorisés, sur la base de la « race $»^{5}$. De plus, parallèlement aux lois et législations, la terreur est utilisée par le régime et elle apparaît comme une nécessité afin de mieux contrôler la population noire et d'affaiblir encore plus sa position .

3. Marc Aiko Zike, La nouvelle Afrique du Sud post apartheid, Paris, L'Harmattan, 2010, p. 14, 16 et 89.

4. Jean-Yves Dhermain, La planète ovale. Dans les coulisses du rugby mondial, Tours, CLD, 2007, p. 148.

5. François-Xavier Fauvelle-Aymar, « Et l'Afrique du Sud inventa l'apartheid », L'Histoire, vol. 306, ח 2 (février 2006), p. 34, 36-37.

6. Marc Aiko Zike, op. cit., p. 19 et 41. 
Des événements sur la scène internationale modifient cependant le climat sud-africain. La fin de la guerre froide amène une perception plus favorable de l'opposition sud-africaine. De plus, le pays est dans une impasse économique avec les différents embargos imposés et il projette une image négative à l'international puisque l'apartheid devient de moins en moins acceptable. Le contexte externe change donc au moment même où, à l'interne, l'arrivée au pouvoir de Frederik De Klerk en août 1989 favorise les pourparlers pour la sortie de l'apartheid'. Les négociations en vue de la libération de Nelson Mandela sont également enclenchées. Le leader de l'ANC est arrêté à l'issue du massacre de Shaperville de 1960 durant lequel des policiers blancs abattent des manifestants noirs. L'ANC est interdit et ses leaders emprisonnés. Mandela est arrêté le 5 août 1962. Avec 26 années passées derrière les barreaux, Mandela est le plus vieux prisonnier politique du monde. Il est libéré définitivement le 11 février 1990. Il appelle dès lors la branche armée de l'ANC à cesser ses activités et, en 1991, la plupart des lois raciales en vigueur depuis des décennies sont abrogées tandis qu'est signé un accord de paix national. Les premières élections démocratiques multiraciales ont lieu au mois d'avril 19948. L'élection de Mandela à la présidence sud-africaine au sein du premier gouvernement démocratique de son histoire jette les bases organisationnelles d'une toute nouvelle nation. Les enjeux sont nombreux et les défis à relever multiples.

Le plus urgent d'entre eux est sans aucun doute la question de la cohabitation raciale. Comment peut-elle se faire alors que la mémoire traumatique de l'apartheid teinte la société de préjugés raciaux? Mandela comprend dès le départ que l'image d'une nation unie est très importante afin de démontrer à l'échelle internationale que la nouvelle Afrique du Sud démocratique est tournée vers l'avenir, unie et pacifiée après les troubles internes, les conflits armés des dernières années et suite à 40 ans d'apartheid.

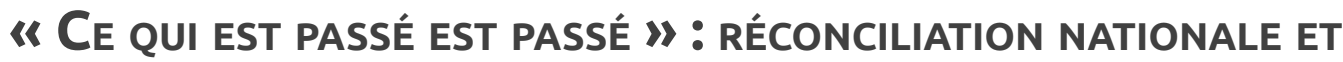 PARDON AUTOUR DE MANDELA}

La tenue de la première élection multiraciale porte au pouvoir le héros de la lutte contre l'apartheid, Nelson Mandela. L'homme politique doit cependant composer avec un pays libéré légalement de l'oppression blanche, mais qui demeure, dans les faits, empreint de préjugés raciaux. L'arrivée du premier président noir de l'histoire du pays enchante la majorité noire, mais inquiète la population blanche : cette dernière craint que Mandela, ce «terroriste ", n'exerce une violence sur la minorité afin de venger les sévices endurés par la population africaine durant l'apartheid. II n'est cependant pas question pour Mandela d'adopter

7. François Fauvelle-Aymar, loc. cit., p. 43-44.

8. Ibid., p. 43-44. 
une politique de confrontation envers les Afrikaners. Au contraire, pour lui il est nécessaire d'opter pour une attitude consensuelle afin de parvenir à une réconciliation raciale pacifique.

\section{Aimer son enNemI : UNE ATtITUdE CONSENSUelLe}

Pour Mandela, le passé est le passé : de ce fait, il est essentiel de pardonner aux Afrikaners et de tendre la main à cet ancien ennemi dans la nouvelle Afrique du Sud démocratique. À travers ses mémoires, Un long chemin vers la liberté, transparaissent son attitude conciliante et son absence de haine envers ceux qui ont été, durant toutes ces années, ses bourreaux. La haine de Mandela est dirigée vers le système de l'apartheid et non pas vers les Blancs. II met en exergue l'importance du rôle des Blancs dans la reconstruction de la nouvelle Afrique du Sud et pense que les chasser serait une erreur et mettrait en péril la fragile nation démocratique.

Il est nécessaire de travailler avec les Blancs et non pas contre eux, de tendre la main vers ceux qui hier étaient des ennemis. Telle est la philosophie politique de Mandela qui s'éloigne d'une attitude de confrontation et se préoccupe de la sécurité de la minorité blanche dans l'Afrique du Sud post apartheid. Aimer son ennemi signifie aussi être un exemple pour la population sud-africaine. En tant que plus vieux prisonnier politique du monde et héros de la lutte antiapartheid, Mandela acquiert une autorité morale qui fait de lui un exemple à suivre. Il réitère son idée de bâtir ensemble la nouvelle Afrique du Sud démocratique. Selon lui, les Afrikaners sont des Sud-Africains au même titre que les Noirs et les deux groupes peuvent trouver leur place dans une nation unifiée et réconciliée, peu importe la couleur de la peau.

C'est un véritable appel à l'unité nationale derrière un but commun, un avenir partagé par les deux populations ${ }^{9}$. En tant qu'exemple pour la population noire, Mandela adopte une politique de gestes symboliques qui vise la réconciliation. Pour ce faire, il utilise des symboles chers aux Afrikaners afin d'opérer un rapprochement. II cite la poète afrikaner Ingrid Jonker et noue des liens avec des figures de l'ancien régime tels que la veuve de l'architecte de l'apartheid Hendrik Verwoerd et le procureur Percy Yutar, celui-là même qui l'a condamné à la prison à perpétuité lors du procès de Rivonia. Finalement, il devient supporter de l'équipe nationale de rugby, symbole du nationalisme afrikaner, se montrant publiquement aux matches du XV sud-africain ${ }^{10}$. Mandela a compris qu'aimer son ennemi, c'est aimer les symboles qu'il chérit. Ainsi, prouve-t-il aux Blancs qu'il n'est pas le « terroriste » que ces derniers redoutent et qu'eux aussi ont leur place dans l'avenir du pays.

9. Nelson Mandela, Un long chemin vers la liberté, Paris, Fayard, 1995, p. 587.

10. Thierry Vircoulon, «La nouvelle Afrique du Sud. Une transformation à géométrie variable », Études, vol. 401, n 12 (décembre 2004), p. 587. 
La réconciliation et le pardon sont des thèmes récurrents dans Invictus et il est possible de noter qu'ils s'articulent autour de la figure de Mandela : c'est lui le véritable artisan de cette réconciliation nationale. II débute cette réconciliation dans son entourage, en rassurant ses fonctionnaires blancs qui anticipent un congédiement avec l'installation d'un pouvoir noir au palais. Devant ses employés blancs rassemblés, Mandela affirme que la couleur de leur peau ou la langue qu'ils parlent ne les disqualifie pas pour travailler avec lui. Le passé est le passé ${ }^{11}$. En acceptant de travailler avec des Blancs, Mandela les maintient non seulement au sein de son administration, mais aussi dans la construction de la nouvelle nation. La même façon de penser pousse Mandela à faire travailler ensemble Noirs et Blancs au sein de son propre personnel de sécurité. De ce fait, à chacune de ses apparitions publiques, entouré à la fois de Noirs et de Blancs, il présente le reflet de la nation arc-en-ciel où la cohabitation est possible et où les deux peuples travaillent ensemble ${ }^{12}$. À travers ces scènes tirées d'Invictus, on remarque que la composition du personnel de Mandela est véritablement utilisée à des fins de démonstration de la possibilité d'une cohabitation noire/blanche. Les Noirs et les Blancs, en tendant vers un but commun tel que la participation à la construction de la nation ou à la protection de l'homme politique le plus important de sa génération, oublient les vieilles discriminations d'autrefois et parviennent à travailler ensemble. Le thème du pardon est mis en avant par Eastwood dans Invictus et est donc présenté comme nécessaire pour parvenir à travailler avec l'ancien ennemi blanc.

\section{LA COMMISSION VÉRITÉ ET RÉCONCILIATION : UN SYMBOLE D'UNIFICATION NATIONALE}

La recherche de la vérité est une étape importante vers une réconciliation entre les Noirs et les Blancs en Afrique du Sud. La commission Vérité et Réconciliation, vue comme le « miracle sud-africain », se veut pacifique et symboliquement réconciliatrice. On auditionne les auteurs de crimes en vue de leur octroyer un pardon moral symbolique. Établie en 1995 et présidée par l'archevêque Desmond Tutu, premier Noir à être nommé à la tête d'un diocèse en Afrique du Sud, la CVR a pour but d'éclaircir les différents crimes commis dans le pays durant la période de l'apartheid. On veut rompre avec l'ancienne culture du silence, favoriser la confession publique et octroyer un pardon symbolique à ceux qui se présentent devant la commission.

Elle s'inscrit dans un contexte de redéfinition et de refondation de la nation sud-africaine. Cette invitation au pardon est destinée à encourager les Sud-Africains à se réconcilier entre eux et à construire une nouvelle nation. Le concept qui guide le mandat de la commission est

11. Clint Eastwood, Invictus, Warner Bross, 2009, 11:04 minutes.

12. Ibid., 14:19 minutes. 
assez unique : on délaisse la justice de vengeance, voire punitive, en optant pour une justice restauratrice et réconciliatrice. De ce fait, on s'inspire grandement de la justice traditionnelle africaine qui laissait toujours place aux compromis et à la recherche d'un consensus dans la prévention et la gestion de conflits. On vise la transformation de la société par ce travail fait auprès des bourreaux et des victimes : c'est une véritable thérapie nationale.

Des critiques sont cependant adressées à cette commission. D'abord, on soulève le fait que si les bourreaux acceptent cette institution, c'est pour échapper aux poursuites judiciaires, donc par intérêt personnel. Ensuite, la CVR a été incapable de traduire les hauts responsables politiques et militaires de l'apartheid qui ont - pour la plupart - refusé de demander l'amnistie. Enfin, la commission ne s'est intéressée qu'à des actes criminels et écarte les aspects ordinaires de la discrimination comme le déplacement forcé des populations noires par exemple ${ }^{13}$.

En outre, autant le gouvernement de De Klerk que celui de l'ANC ont refusé de faire leur mea culpa et de reconnaître leurs fautes devant la commission ${ }^{14}$. On peut donc dire que la réussite de la commission doit être nuancée sur certains points. Malgré tout, la portée symbolique de la CVR reste très forte. Dès lors, il s'agit de voir comment Mandela, de par son attitude consensuelle, va opérer la réconciliation raciale dans son pays autour des Springboks, l'équipe de rugby sud-africaine, symbole de l'oppression blanche et de l'apartheid.

\section{One team, one country: les Springboks comme Vecteur D'IDENTITÉ ET DE FIERTÉ NATIONALE}

Des divers symboles chers à la population afrikaner, c'est l'équipe nationale de rugby qui retient l'attention de Mandela comme vecteur d'identité et de fierté nationale. Cependant, pourquoi ce sport est-il justement choisi par Mandela comme base de la réconciliation? Certes, c'est le sport de prédilection des Afrikaners, mais au-delà de cet aspect, on peut dire que le rugby, par sa culture sportive et les valeurs morales véhiculées, trouve un écho dans la situation sud-africaine.

Les origines du rugby en Afrique du SUd

Le rugby sud-africain est une importation britannique. C'est dans les années 1870 qu'il se développe, avec l'arrivée des immigrants attirés par la découverte des richesses du soussol et la création d'établissements scolaires. Principalement d'origine britannique, ces immi-

13. Laetitia Bucaille, "Vérité et réconciliation en Afrique du Sud. Une mutation politique et sociale », Politique étrangère, $n^{\circ} 2$ (été 2007), p. 319.

14. Thierry Vircoulon, loc. cit., p. 597. 
grants amènent avec eux leur passion du ballon ovale et, bientôt, quelques sociétés de rugby s'installent à travers le territoire. Progressivement, le rugby des Anglais remplace le football de Gog, créé par le révérend chanoine George Olgivie, responsable du Diocesan College de Rondebosch et qui est la première forme de rugby sud-africaine. Peu à peu, les établissements scolaires prestigieux tels que le Hilton College et le Dale College abandonnent le football de Gog pour le rugby. En 1889 est créé le South African Rugby Board (S.A.R.B) qui a pour mission de gérer ces différentes sociétés de rugby à travers le pays. Au-delà de cette gestion se cache également la volonté de surveiller ce pays vaste, morcelé en régions et sa population afrikaner. La propagation de ce sport est aussi favorisée par les tournées organisées par les Britanniques et d'autres équipes européennes. Les Afrikaners sont rapidement attirés par ce jeu viril et ils y participent massivement : en 1880, sur les 19 noms de la première équipe de Worcester, trois seulement sont britanniques ${ }^{15}$. Mais la véritable popularité du rugby débute à la fin du XIXe et au début du XXe siècle. Les Afrikaners, ces Sud-Africains descendants des Hollandais, choisissent le rugby comme exutoire contre l'oppression britannique. Autrefois membres de la colonie néerlandaise conquise par l'Empire britannique, les Boers voient leur tentative d'indépendance réprimée dans le sang par les Anglais. La mémoire de cet événement traumatique est toujours très vive chez les Afrikaners, à la sortie du conflit en 1910 lorsque le roi Édouard VII décide de fédérer les colonies du Cap, du Transvaal, d'Orange et du Natal au sein de l'Union sud-africaine ${ }^{16}$. Le rugby devient alors un véritable moyen pour les Afrikaners de canaliser leur rage et leur déception : jouer au rugby représente le fait d'entrer dans un rapport de force avec l'oppresseur britannique.

\section{UN SPORT DE COMBAT : VALEURS MORALES ET CULTURE SPORTIVE}

Le rugby séduit les Afrikaners par les valeurs morales qu'il véhicule : le sacrifice, le goût de la discipline et du collectif sont des aspects très attrayants pour cette communauté jadis oppressée par les Britanniques. Installé en Afrique du Sud, en tant que symbole afrikaner, le rugby est probablement le sport le plus viril qui existe. L'historien Pierre Chaix, dans Les jeux troubles du rugby sud-africain écrit que «le rugby est un sport de combat collectif rude, dur, où il faut s'imposer pour "survivre ${ }^{17}$. Cette démonstration de force et de virilité des joueurs de même que les victoires et titres remportés par l'équipe reflètent certes la force de l'équipe, mais également la force de la nation en tant que telle. Le couple équipe/nation s'observe à plusieurs reprises dans l'œuvre d'Eastwood Invictus et un parallèle intéressant peut être fait entre la reconstruction de la nation et celle de l'équipe nationale. Car, pour favoriser une image puissante à l'international, on doit compter sur une équipe récoltant les succès. Or, depuis l'apartheid, les Springboks connaissent des jours difficiles et les victoires se font

15. Jean-Pierre Bodis, Histoire mondiale du rugby, Bibliothèque historique Privat, Toulouse, 1987, p. 114-121. 16. Jean-Yves Dhermain, op. cit., p. 147.

17. Ibid. 
rares. Victimes d'une mauvaise presse et de l'embargo sportif, ils sont, à la sortie de l'ancien régime, à la recherche d'une crédibilité au sein de la planète ovale. Si Pienaar désire reconstruire son équipe afin de rétablir la gloire d'antan, Mandela veut, pour sa part, reconstruire I'Afrique du Sud. Les deux poursuivent le même but et s'influencent mutuellement. Avoir une équipe victorieuse est très important pour démontrer la réussite de la nation arc-en-ciel : c'est pourquoi la pression est forte sur les Springboks. Ils ne sont plus seulement une équipe de rugby, il y a un enjeu plus grand encore derrière la victoire. Outre la grande force physique et l'endurance nécessaires pour atteindre ce but, l'importance du groupe et de la solidarité entre les joueurs est primordiale : il n'y a pas d'équipe efficace sans cela.

Cette solidarité se créé sur le terrain, avec le corps à corps et l'esprit de combat, mais aussi à l'extérieur du terrain dans le mode de vie et l'entraide entre les joueurs qui renforce l'idée selon laquelle le rugby est définitivement une grande famille. Une confiance absolue doit également régner entre les membres de l'équipe, due à la rudesse de l'affrontement physique et à l'importance de ne pas plier devant l'adversaire. La réussite collective dépend du don de soi. On voit donc qu'à travers les valeurs morales transmises par la pratique du ballon ovale, des liens s'établissent avec la nécessaire reconstruction nationale. La réussite dépend du groupe, de la solidarité, de la cohésion unissant les joueurs. À ce titre, on comprend qu'un travail d'équipe, une solidarité et une confiance doivent nécessairement s'établir entre les Noirs et les Blancs.

\section{L'HYMNE NATIONAL SUD-AFRICAIN : RÉUNION AUTOUR DU CHANT PATRIOTIQUE ET DE LA RELIGION}

Le rugby est un sport où l'hymne national revêt une importance significative. L'image de ces hommes debout devant leurs partisans se tenant bras dessous bras en chantant l'hymne national de leur pays, telle que présentée dans Invictus, cristallise le sentiment d'appartenance et l'identité nationale. C'est la raison pour laquelle les Springboks doivent nécessairement apprendre le nouvel hymne national suite à l'abolition de l'apartheid : un hymne qui comprend certaines parties en langues africaines. Initialement irrecevable pour les joueurs des Boks, ces derniers prennent rapidement conscience de l'enjeu national que l'équipe représente dorénavant. En tant que symbole de la réconciliation, les Springboks acceptent et apprennent le nouveau chant, Nkosi Sikelel' iAfrika. L'image de ces hommes forts, virils et blancs qui chantent leur hymne, incluant les parties en langues africaines dont ils ne comprennent pas le sens, contribue beaucoup à la réconciliation raciale ${ }^{18}$.

Le chant puissant sud-africain de Nkosi Sikelel' iAfrika constitue une sorte de réparation mémorielle. II représente pour la population beaucoup plus qu'un simple chant : il porte une symbolique très forte pour ce pays dont la mémoire de l'apartheid demeure vive et trauma-

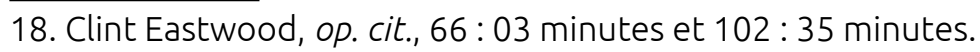


tique. Ce chant de libération essaime au-delà de l'Afrique du Sud, transportant un message d'unité et exhortant à agir moralement et spirituellement au nom du continent africain. II s'agit véritablement d'un chant de bénédiction. En 1897, Enroch Sontonga, une enseignante d'une école méthodiste à Johannesburg compose Nkosi dans le cadre d'un cours de préparation de chanson pour les élèves de l'école.

La chanson originale combine à la fois un hymne harmonique méthodiste et un chant de louange africain qui inclue les concepts de rédemption et de bénédiction africaine. En 1925, I'ANC adopte officiellement Noksi en tant qu'hymne. Depuis 1994, Nkosi Sikelel' iAfrika est I'hymne national de l'Afrique du Sud et est officiellement publié en plusieurs langues : afrikaans, xhosa, zoulou, sotho, anglais. II a cependant été abrégé ${ }^{19}$ et on y mixe l'ancien hymne afrikaner de l'apartheid, Die Stem van Suid Afrika ${ }^{20}$. Le mariage entre les deux chants constitue une sorte de compromis. Le mélange ethnique et religieux de la pièce fait en sorte que tous les peuples composant l'Afrique du Sud se sentent réunis par l'hymne. Donc, de par la culture sportive et les valeurs morales de victoire, de solidarité du groupe et de don de soi qu'il véhicule, le rugby devient, de tous les symboles afrikaners, la base de la réconciliation raciale imaginée par Mandela.

\section{BÂTIR LA NATION AVEC DES BRIQUES ENVELOPPÉES DE VERT ET OR}

Sans l'action de Mandela, les Springboks n'existeraient plus aujourd'hui. Le gouvernement de I'ANC décide en 1994 de faire table rase des différents symboles associés à l'apartheid dont le drapeau et le Die Stem, l'hymne national en langue afrikaans. Les Boks sont également menacés d'élimination par le nouveau pouvoir noir qui voit dans l'équipe le symbole de l'oppression blanche. Le Conseil des sports souhaite donc remplacer l'emblème de l'équipe, l'antilope bondissante, par celui de la protea, une fleur spécifique au pays et plus représentative de la nation arc-en-ciel selon la nouvelle administration. Ce faisant, le gouvernement désire rompre avec le passé traumatique. Par contre, Mandela s'oppose catégoriquement à la destitution de l'équipe qu'il considère comme élément du patrimoine sud-africain et, de ce fait, propriété de la nation entière autant blanche que noire ${ }^{21}$.

Clint Eastwood présente d'ailleurs une scène sur ce sujet dans Invictus. Dans son allocution devant le Conseil des sports, Mandela demande le rétablissement des Springboks. II affırme que les Blancs ne sont plus des ennemis, mais des compatriotes sud-africains au sein d'une

19. Bennetta Jules-Rosette et David B. Coplan, « « Nkosi Sikelel' iAfrika» From Independent Spirit to Political Mobilization », Cahier d'Études africaines, vol. 173-174, nº 1 (2004), p. 435.

20. Julien Migozzi, «Le rugby en Afrique du Sud face au défi de la transformation : jeu de pouvoir, outil de développement et force symbolique », Les Cahiers d'Outre-Mer, n² 250 (avril-juin 2010), p. 261.

21. Jean-Yves Dhermain, op. cit., p. 145. 
démocratie. Leur enlever les Springboks, c'est entrer dans une confrontation avec eux, c'est la confirmation de leurs craintes envers le pouvoir noir. Pour Mandela, la population noire doit étonner les Blancs par sa retenue et sa compassion.

Ce n'est pas le moment de s'adonner à de petites vengeances : il faut bâtir la nation, et ce, même si les briques sont enveloppées dans du Vert et Or22, les couleurs officielles du XV sud-africain. La métaphore utilisée démontre le lien existant entre la construction nationale et l'équipe des Springboks. Cette dernière devient la fondation sur laquelle le chantier de la réconciliation nationale se met en branle. Mandela ne cherche donc pas la confrontation avec ses compatriotes blancs. Le but n'est pas de renforcer le cycle de la peur entre les Noirs et les Blancs, mais bien d'y mettre un terme.

Pauvreté, Jeunesse et Rugby : une forme de coopération RaCiale

Le rôle du rugby est très important dans Invictus et le réalisateur reconstruit la réconciliation telle qu'elle avait été envisagée par Mandela. Les Springboks sud-africains deviennent bien plus qu'une simple équipe de rugby durant l'année précédant la Coupe du monde de 1995. Si la réconciliation raciale s'opère autour du ballon ovale comme le veut le président, comment faire accepter à la population noire une équipe et un sport qu'elle a toujours considéré comme le symbole de l'apartheid? Le film Invictus présente une scène éloquente sur la manière dont Mandela va réussir à faire connaître, accepter et aimer le rugby à la population noire. Sur sa demande, les joueurs des Boks se rendent dans un township pour offrir un atelier sportif d'une journée sur le rugby. L'équipe, accompagnée de journalistes et de caméramans, est filmée alors qu'elle joue et enseigne à la jeunesse sud-africaine noire les rudiments de base du ballon ovale. Les images recueillies sont retransmises le soir même à la télévision ${ }^{23}$.

La symbolique de cette journée est très forte : des Blancs enseignent à de jeunes Noirs défavorisés un sport typiquement afrikaner qu'ils ont toujours profondément haï. Les images captées montrent qu'une coopération raciale est possible entre les deux groupes : non seulement on peut travailler ensemble, en équipe, mais on peut aussi rire, avoir du plaisir de façon commune, et donc vivre ensemble. Par cette scène, on constate vraiment qu'à travers le rugby s'opère un rapprochement racial, orchestré par Mandela, le véritable artisan de cette réconciliation.

22. Clint Eastwood, op. cit., 28:51 minutes.

23. Ibid., 57:28 minutes. 
Pour les partisans, la valorisation du maillot d'une équipe sportive et de ses couleurs contribue au renforcement des comportements identitaires ${ }^{24}$. Se retrouver en tant que supporter derrière la même équipe constitue, pour un groupe donné, un important vecteur identitaire et donne un sentiment d'appartenance fort. Par contre, on voit dans Invictus qu'un maillot des Springboks est automatiquement synonyme de l'apartheid pour la population noire lorsqu'un jeune noir défavorisé refuse d'adopter un maillot des Boks : il préfère porter ses haillons plutôt qu'un authentique maillot ${ }^{25}$. Cette haine des Noirs pour l'équipe nationale se manifeste également durant les matchs amicaux de rugby, dont le match préparatoire contre l'Angleterre, prélude à la Coupe du monde que l'Afrique du Sud devrait accueillir.

Tous les Blancs prennent parti pour l'Afrique du Sud tandis que tous les Noirs encouragent l'adversaire, uniquement dans le but d'énerver et d'irriter le public afrikaner. Ils trouvent dans le fait d'encourager les Britanniques, oppresseurs traditionnels des Afrikaners et de huer les Springboks, une certaine revanche prise sur leur propre oppresseur ${ }^{26}$. Si au début du long métrage, on voit clairement les divisions entre les spectateurs qui assistent aux matchs, le dénouement montre l'image d'un pays uni derrière son équipe nationale. II faut en effet souligner que pour en arriver là un important travail est effectué, à travers la figure de Nelson Mandela.

Celui-ci assiste aux matchs, salue la foule et serre la main des joueurs : il se présente publiquement en tant que partisan des Springboks ${ }^{27}$. Le jour de la finale, c'est un Mandela souriant et excité qui va saluer la foule et les rugbymen, arborant le maillot numéro 6 du capitaine François Pienaar et une casquette à l'effigie des Springboks ${ }^{28}$. Un Noir qui porte le maillot et les couleurs d'une équipe qui fut jadis la vitrine de la réussite du système de l'apartheid est une image très frappante. Ce faisant, Mandela montre l'exemple à la population noire : I'heure est au pardon et à la réconciliation et pour ce faire, il faut être uni derrière l'équipe nationale.

C'est donc en adoptant une attitude consensuelle que Mandela orchestre la réconciliation à travers l'équipe de rugby sud-africaine des Springboks qu'il soutient fermement. Ce moment fort où Pienaar reçoit la Coupe des mains de Mandela, dans un stade en liesse rempli de

24. Sébastien Darbon, dir., Rugby d'ici. Une manière d'être au monde, Paris, Autrement, 1999, p. 42.

25. Clint Eastwood, op. cit., 27:46 minutes.

26. Ibid., 21:04 minutes.

27. Ibid., 19:00 minutes.

28. Ibid., 99:59 minutes. 
partisans blancs et noirs, symbolise véritablement la réussite d'une réconciliation raciale et présente une image unifiée de l'Afrique du Sud sur la scène internationale ${ }^{29}$.

\section{Conclusion}

La réconciliation raciale en Afrique du Sud s'articule donc bel et bien autour de Nelson Mandela. À travers une attitude consensuelle vis-à-vis de son ennemi, Mandela pardonne et inclut la minorité blanche dans la reconstruction et l'avenir du pays. La réconciliation telle qu'imaginée par Mandela s'orchestre à travers la captation d'un élément important du nationalisme afrikaner : les Springboks, l'équipe de rugby de l'Afrique du Sud. La reconstruction de la nation se déroule en parallèle à la remise sur pied de l'équipe nationale de rugby, déchue depuis l'instauration de l'embargo sportif contre l'Afrique du Sud. La nécessité de gagner est primordiale pour le sentiment d'unité nationale, mais aussi pour montrer l'image d'un pays victorieux et dorénavant unifié sur la scène internationale. Cependant, si au terme d'Invictus on a le sentiment que la réconciliation raciale est un succès, il n'en est pas véritablement ainsi dans les faits.

L'Afrique du Sud demeure marquée par des préjugés raciaux, et ce, même au sein des Springboks. Certains cas de racisme sont répertoriés depuis quelques années. En 2003, l'avant blanc Géo Cronje refuse d'avoir son équipier noir Quiton Davids comme compagnon de chambre. L'attaché de presse des Springboks démissionne de son poste et dénonce les préjugés raciaux qui perdurent dans le rugby sud-africain. Notons malgré tout l'année 2006 comme importante puisque, pour la première fois, 14 joueurs non blancs font partie de la sélection ${ }^{30}$. Si la réconciliation telle que vue dans Invictus est présentée de façon relativement idéalisée, il n'en demeure pas moins qu'elle est uniquement symbolique et destinée à la création d'une certaine image sud-africaine sur l'échiquier mondial. Des troubles internes subsistent encore aujourd'hui, résultat de plus de quarante années de régime discriminatoire. 1994 représente un départ pour la jeune démocratie sud-africaine. Dans les années suivantes, le développement de la nation arc-en-ciel occupe une grande place dans l'actualité internationale. À travers cette couverture journalistique et le traitement de l'information, il est certainement possible de relever la vision qu'a la communauté internationale de cette nouvelle Afrique du Sud post apartheid, égalitaire et démocratique. Parallèlement à cela, on peut également observer la dimension mémorielle qui se dégage de cette actualité et déterminer la mémoire de l'apartheid qui est véhiculée.

29. Ibid., 125:12 minutes.

30. Jean-Yves Dhermain, op. cit., p. 160-161. 


\section{SOURCES ET BIBLIOGRAPHIE}

AIKO ZIKE, Marc. La nouvelle Afrique du Sud post apartheid. Paris, L'Harmattan, 2010, 115 p.

BODIS, Jean-Pierre. Histoire mondiale du rugby. Toulouse, Bibliothèque historique Privat, 1987, 415 p.

BUCAILLE, Laetitia. «Vérité et réconciliation en Afrique du Sud. Une mutation politique et sociale ». Politique étrangère, $n^{\circ} 2$ (2007), p. 313-325.

CORRIGALL, Marie. « Mandela revu et corrigé dans Invictus ». Courrier international, (12 janvier 2010), [en ligne] http://www.courrierinternational.com/article/2010/01/12/mandela-revu-et-corrige-dans-invictus, page consultée le 9 mai 2013.

DARBON, Sébastien, dir. Rugby d'ici. Une manière d'être au monde. Paris, Autrement, 1999, 221 p.

DHERMAIN, Jean-Yves. La planète ovale. Dans les coulisses du rugby mondial. Tours, CLD, 2007, 297 p.

EASTWOOD, Clint. Invictus. Film, Warner Bross, 2009, 2 h 10 min.

FAUVELLE-AYMAR, François-Xavier. «Et l'Afrique du Sud inventa l'apartheid ». L'Histoire, vol. 306, nº 2 (février 2006), p. 34-45.

JULES-ROSETTE, Bennetta et David B. COPLAN. « « Nkosi Sikelel' iAfrika” From Independent Spirit to Political Mobilization ». Cahiers d'Études africaines, vol. 173-174, n 1 (2004), p. 343-367.

MANDELA, Nelson. Un long chemin vers la liberté. Paris, Fayard, 1995, 649 p.

MANDELBAUM, Jacques. "Invictus : réconciliation raciale sur une pelouse de rugby ». Le Monde, (12 janvier 2010), [en ligne] http://www.lemonde.fr/cinema/article/2010/01/12/invictus-reconciliation-raciale-sur-une-pelouse-de-rugby_1290640_3476.html, page consultée le 9 mai 2013.

MIGOZZI, Julien. « Le rugby en Afrique du Sud face au défi de la transformation : jeu de pouvoir, outil de développement et force symbolique », Les Cahiers d'Outre-Mer, n 250 (avril-juin 2010), p. 253274.

VIRCOULON, Thierry. «La nouvelle Afrique du Sud. Une transformation à géométrie variable». Études, vol. 401 (décembre 2004), p. 585-600. 